\title{
LES CLIENTS DES SERVICES D'ESCORTE TELS QUE PERÇUS PAR DES FEMMES OFFRANTS CES SERVICES
}

\section{Jacqueline Comte \\ Université Laval}

La discussion de projets de loi criminalisant l'achat des services sexuels constitue un sujet brûlant d'actualité dans plusieurs pays. Le Canada vient d'adopter une telle loi. Pourtant, un bon nombre d'études indiquent que la plupart des clients agissent de manière civilisée envers la personne travailleuse du sexe, et que seule une infime minorité de clients présentent des comportements violents. Interviewant 16 femmes offrant des services d'escorte quant à leur expérience de sexualité dans le cadre du travail, celles-ci ont beaucoup discuté des besoins qu'elles percevaient chez leurs clients, de même que des attitudes de ces derniers envers elles. Les participantes décrivent ceux-ci comme étant généralement respectueux, la plupart recherchant un contact chaleureux avec elles et agissant en conséquence. En outre, les clients qui ne recherchent qu'une expérience sexuelle tendent à être également respectueux envers l'escorte. Par contre, certains clients présentent une attitude ou un comportement désagréable. Quelques participantes ont également eu affaire à un client violent au cours de leur parcours comme escorte. La plupart des participantes étaient en mesure de choisir leurs clients, ce qui leur a permis de surtout rencontrer des clients aimables et qui, à l'occasion, leur procure également du plaisir sexuel. Par contre, les participantes plus vulnérables et moins capables de choisir et de maintenir leurs limites ont rencontré plus de clients désagréables ou agressifs.

Mots-clés: Travail du sexe; client; comportement; intimité; violence.

The discussion of bills criminalizing the purchase of sexual services is a very topical subject in many countries, and Canada has just passed such legislation. However, many studies indicate that most clients act in a civilized manner towards a sex worker, whereas only a tiny minority exhibits violent behavior. In the course of interviewing 16 women offering escort services about their sexual experience in the context of their work, the participants talked a lot about the needs they sense their clients have as well as their clients' attitude towards them. They described their clients as polite for the most part, with the majority seeking a warm contact with them and acting accordingly. In addition, those clients who are only looking for a sexual encounter tend to be equally respectful towards the escort. By contrast, certain clients exhibit a bad attitude or rude behavior. Some participants also had to deal with a violent client during their career as escorts. Most of them were able to choose their clients, allowing them to meet nice ones who occasionally give them sexual pleasure too. On the other hand, those participants who are more vulnerable and less able to choose and maintain their limits have met more unpleasant or aggressive clients.

Keywords: sex workers; clients; behavior; intimacy; violence. 


\section{Une criminalisation des clients?}

Un mouvement abolitionniste exigeant la criminalisation des clients se fait de plus en plus entendre dans le monde politique de plusieurs pays occidentaux. Au Canada, lors des débats entourant le projet de loi du gouvernement conservateur concernant le travail du sexe, ce mouvement a été représenté par la Coalition des femmes pour l'abolition de la prostitution, un regroupement canadien de sept organismes. Selon ceux-ci, la prostitution ne peut en aucune manière être considérée comme un travail légitime puisqu'elle constitue une violence envers les femmes en les réduisant à l'état d'objets destinés à être économiquement et sexuellement exploités par les hommes. De plus, étant violence, la prostitution entraine dans son sillon tous les autres types de violence dont les hommes sont capables. Il est donc inconcevable, toujours selon la coalition, qu'une femme puisse consentir à se prostituer car personne n'accepterait de plein gré d'être brutalisée à chaque jour tout en se sachant constamment à risque d'être tuée par un souteneur ou un client. La prostitution exploitant les femmes et les enfants, elle est intrinsèquement liée à la traite des femmes et des enfants pour fins d'exploitation sexuelle et c'est seulement en abolissant la prostitution qu'on pourra éliminer cette traite. Par ailleurs, la prostitution existe parce qu'il y a une demande de la part d'hommes «prostitueurs », c'est-à-dire de clients qui prostituent la femme en l'utilisant pour satisfaire leurs propres pulsions sexuelles, usant dans un même temps de coercition et de violence. Criminaliser les clients réduirait la demande et serait un bon pas dans la direction de l'idéal de disparition de la prostitution, laquelle est nécessaire afin de parvenir à l'égalité des femmes et des hommes. (Barry, 1979, 1995; Coalition des femmes pour l'abolition de la prostitution, 2013; Dworkin, 1979, 1993; Farley \& Barkan, 1998; Farley, Cotton, Lynne, Zumbeck, Spiwak, Reyes, Alvarez \& Sezgin, 2004; Farley, 2003, 2004, 2005; Farley, Bindel \& Golding, 2009; Hugues, 2005; Jeffreys, 1997, 2008, 2009; MacKinnon, 1985; Poulin, 2004; Raphael \& Shapiro, 2002, 2004; Raymond, 2003, 2004). Forte de ce discours, la Suède, la Norvège et l'Islande ont ainsi criminalisé les clients en argumentant que les femmes prostituées sont nécessairement victimes de ces derniers (Waltman, 2011). Suivant cet exemple, des politiciens et politiciennes d'autres pays, dont la France, l'Angleterre et l'Allemagne, tentent de faire adopter des lois semblables. La Chambre des Communes et le Sénat du Canada, de leur côté, ont voté en novembre 2014 une «Loi sur la protection des collectivités et des personnes exploitées »(C-36), laquelle criminalise les clients. Ce mouvement grandissant de criminalisation des clients pose la question de qui est ce client, au juste? Il devient donc important de disposer de données empiriques solides sur le client porté maintenant à l'avant-scène. Cet article vise dès lors à ajouter aux données existantes, lesquelles décrivent le client de manière beaucoup plus nuancée que ne le font les écrits abolitionnistes.

\section{Les données empiriques sur les clients se multiplient}

Depuis une quinzaine d'années, de plus en plus d'études s'intéressent aux clients, à leurs motivations à acheter des services sexuels et à leurs attitudes face aux travailleuses du sexe (par ex., Bernstein, 2007; Di Nicola, Cauduro, Lombardi, \& Ruspini, 2009; Holt \& Blevins, 2007; Jeffrey \& MacDonald, 2006; Jordan, 1997; Joseph \& Black, 2012; Katsulis, 2010; Kinnell, 2006; Klein, Kennedy, \& Gorzalka, 2009; Lowman \& Atchison, 2006; Milrod \& Weitzer, 2012; Milrod \& Monto, 2012; Monto, 2000a, 2000b, 2004, 2010; Monto \& Hotaling, 2001; Orchard, Farr, Macphail, Wender, \& Young, D. 2013; Pitts, Smith, Grierson, O'Brien, \& Misson, 2004; Prasad, 1999; Sanders, 2008; Sharp \& Earle, 2002; Xantidis \& McCabe, 2000). La plupart de ces travaux 
indiquent qu'en regard des données sociodémographiques, les clients ne sont nullement différents des non-consommateurs de services sexuels. Les travaux de Monto et de ses collaborateurs (Monto, 2000a, 2000b, 2004, 2010; Monto \& Hotaling, 2001; Monto \& McRee, 2005; Monto \& Milrod, 2013) ont, quant à eux, identifié de petites différences, "en degré et non en qualité » (Monto \& McRee, 2005, p. 505, ma traduction) entre les hommes arrêtés alors qu'ils tentaient d'obtenir les services d'une prostituée de rue et les hommes d'un échantillon national. D'une part, les clients arrêtés sont proportionnellement plus souvent célibataires ou moins heureux dans leur mariage et, d'autre part, ils présentent plus souvent des attitudes sexuelles plus libérales.

Une personne peut avoir recours aux services sexuels tarifés pour différentes raisons, lesquelles peuvent se regrouper en deux grandes catégories de motivations. Certains clients sont en quête de variété et recherchent d'abord et avant tout l'excitation sexuelle ou la satisfaction d'un fantasme; ils tendront ainsi à voir une travailleuse du sexe différente à chaque rencontre. D'autres, par contre, préfèrent rencontrer toujours la même - ou les mêmes - travailleuses du sexe et construire avec elles une relation de nature assez semblable à ce qui se passe avec une maîtresse. Ils préfèrent la familiarité et la sécurité émotionnelle et sexuelle d'un lien nourri dans une continuité, et veulent une relation partageant plusieurs caractéristiques avec celle pouvant se développer dans une relation romantique conventionnelle. Ils recherchent un lien d'intimité interpersonnelle et émotionnelle avec la travailleuse du sexe, à travers lequel la sexualité a la possibilité de s'exprimer par le biais d'un échange de plaisirs conduisant à une satisfaction sexuelle mutuelle. Le lien d'intimité se construit en outre à travers l'échange réciproque d'informations personnelles sur sa vie, la conversation et l'échange d'idées, lequel partage se fait pendant les rencontres, mais aussi, dans certains cas, entre les visites par le biais de courriels ou d'appels téléphoniques. Le lien affectif est ainsi ressenti comme un véritable lien d'amitié, lequel demeure toutefois généralement circonscrit à la nature professionnelle de la relation (Clouet, 2008; Lucas, 2005; Jeffrey \& Macdonald, 2006; Lever \& Dolnick, 2010; Milrod \& Monto, 2012; Milrod \& Weitzer, 2012; Sanders, 2008; WelzerLang, Barbosa, \& Mathieu, 1994). Bernstein $(2007,2013)$ a proposé le terme authenticité limitée «bounded authenticity» afin de rendre compte de ce lien qui se construit entre le client et l'escorte qui le reçoit.

Quelle que soit la motivation première (excitation sexuelle pure ou désir d'intimité émotionnelle à travers un échange sexuel), il semble que la plupart des clients perçoivent le sexe tarifé, « non pas comme une pratique compensatoire, [...] ni même comme une substitution pour [l']absence [de sexe], mais plutôt comme un choix souvent préférable » (Serughetti, 2012, p.45; ma traduction, italiques originaux). Ils apprécient pouvoir compter sur une entente claire concernant la nature de la rencontre, ainsi que sur le fait que la relation, étant professionnelle et payée, puisse ne pas déborder dans la vie personnelle (Bernstein, 2013; Prasad, 1999). Joseph \& Black (2012) ont également noté, à la suite d'une étude quantitative des profils de clients, que « la marchandisation du sexe n'est pas inextricablement liée à des perceptions négatives envers les femmes [et qu'il nous faut] déconstruire le lien essentialiste entre la consommation du travail du sexe et le désir qu'aurait ces hommes d'exercer une domination sur les femmes » (p. 503, ma traduction) puisque ce ne sont pas tous les clients qui présentent une attitude inacceptable envers les travailleuses du sexe. Au contraire, la très grande majorité des clients agissent correctement vis-à-vis de celles-ci et la violence subie par les travailleuses du sexe est le fait d'un très petit nombre d'hommes violents (Kinnell, 2006; Klein et al, 2009; Jeffrey \& MacDonald, 2006; Lowman \& Atchison, 2006; Monto, 2000b, 2004, 2010; Oerton \& Phoenix, 2001; Parent \& Bruckert, 2005; Sanders, 2008). Il arrive 
toutefois que des clients, par ailleurs non violents, adoptent un comportement agressif en réaction à un comportement vicitimisant de la part de la travailleuse du sexe, celle-ci ne donnant pas le service pour lequel elle s'est fait payer ou lui volant un bien. Holt \& Blevins (2007), Jeffrey \& MacDonald (2006) et Jordan (1997) ont également mentionné la présence, dans le travail du sexe, de situations d'exploitation ou de comportement violent de la part de certaines personnes travailleuses du sexe envers des clients. Selon Lowman \& Atchison (2006), la violence a donc cours des deux côtés et elle se nourrit de la violence subie.

\section{Notre étude}

Cet article prend appui sur des données que nous avons colligées dans le cadre d'une recherche dont le but était de comprendre comment s'organise et se construit l'expérience de sexualité chez des travailleuses du sexe. Nous avons rencontré 16 femmes offrant ou ayant offert des services d'escorte dans le cadre de deux entrevues semi-dirigées d'environ 90 minutes chacune, afin d'explorer cette expérience en profondeur. Invitées à témoigner de leur expérience de la sexualité avec les clients, les participantes ont également discuté de la perception qu'elles avaient des attitudes et des besoins de leurs clients. Ce sont ces résultats que nous présentons dans le cadre de cet article.

Notre échantillonnage en est un dit «boule de neige ». Les premières entrevues ont été réalisées avec des membres de l'organisme Stella situé à Montréal, ou de l'organisme Projet Intervention Prostitution Québec situé à Québec, et ces premières participantes ont parlé de notre recherche à leurs collègues, les invitant à participer. Nous avons également contacté des escortes et des agences d'escorte s'annonçant sur des sites internet. Toutefois, nous n'avons obtenu aucune réponse de leur part. Considérant le nombre assez élevé de sollicitations, le taux de participation s'avère très faible, ce qui s'explique par le sujet de l'étude (l'expérience de sexualité) et la demande de participer à deux entrevues. Ainsi, nous avons surtout rencontré des femmes à l'aise d'offrir des services d'escorte et à l'aise de parler de leur sexualité. Nous avons entrepris l'analyse des données à l'aide de la méthodologie de théorisation ancrée, de manière à demeurer le plus près possible des données recueillies.

Les participantes étaient âgées de 21 à 54 ans au moment de l'entrevue. Trois d'entre elles ont fait leurs débuts dans le travail du sexe alors qu'elles étaient encore mineures, les autres participantes étant âgées de 18 à 45 ans, pour une moyenne d'âge d'entrée de 24 ans. Elles avaient de 1 an à 16 ans d'expérience dans le travail du sexe, pour une moyenne de 6,9 ans et de 3 mois à 16 ans d'expérience dans l'offre de services d'escorte, pour une moyenne de 4,6 ans. Deux ont quitté l'industrie du sexe parce qu'elles étaient en couple, et une autre parce qu'elle avait obtenu une bourse d'études. Seules deux des participantes, alors mineures, n'avaient pas complété leur scolarité secondaire et ne possédaient aucune expérience de travail antérieure à l'entrée dans l'industrie du sexe. La moitié possédait en outre un diplôme d'études collégiales et quelques-unes étudiaient à l'université. Considérant leur besoin d'un emploi rémunérateur et différentes possibilités s'offrant à elles, la moitié d'entre elles ont choisi d'entrer dans l'industrie du sexe afin d'explorer certains aspects de leur sexualité, alors que, pour deux autres, c'est le plaisir de rencontrer et de connaître différentes personnes dans leur intimité qui a été l'élément motivateur. Par contre, pour un autre tiers des participantes, le travail du sexe est vécu de façon ambivalente, car il est à la fois source de gratifications (se sentir belle et valorisée) et source de malaises (se sentir honteuse de s'engager 
dans une prestation de sexualité rémunérée). Quant à leur parcours dans l'industrie du sexe, la moitié des participantes ont commencé par la danse ou le massage érotique, et plus du tiers ont acquis de l'expérience dans plus de deux types de travail du sexe. En outre, 14 participantes sur 16 ont offert des services d'escorte en tant qu'indépendantes, 10 d'entre elles ayant d'abord travaillé dans des agences pour continuer ensuite en gérant elles-mêmes leurs activités. Il s'agit donc d'un échantillon regroupant essentiellement des femmes provenant de la classe moyenne et possédant des ressources personnelles qui leur auraient permis d'occuper un emploi dans un autre secteur économique, si elles l'avaient souhaité (Comte, à paraître).

\section{Des résultats qui démystifient l'image du client}

\section{a) La quête de services comme réponse à des besoins sexuels et d'intimité affective}

Ainsi que l'expliquent nos participantes, les hommes ayant recours à leurs services d'escorte s'attendent à ce qu'elles répondent à certains de leurs besoins ou désirs sexuels mais, souvent, ils espèrent également combler certains de leurs besoins affectifs. Parfois même, le client donne la priorité aux besoins affectifs, l'activité sexuelle apparaissant alors comme une justification à la rencontre. Par ailleurs, les clients se trouvent dans une grande variété de situations quant à leur statut de couple; ainsi existe-il une variété de raisons sous-jacentes à la quête de services. Chez les célibataires, certains sont simplement trop timides et ne parviennent pas à entrer en relation avec une femme, quelques-uns sont handicapés physiquement et sont par conséquent très peu attirants, d'autres ont pleinement confiance en eux et passablement de «sex appeal» mais préfèrent demeurer célibataires, alors que d'autres encore cherchent à combler un peu du vide vécu entre deux relations. Chez les hommes en couple, il y a ceux dont le couple va mal, ceux qui aiment leur conjointe mais ne peuvent pas avoir des activités sexuelles avec elle soit parce qu'elle ne les souhaite pas ou parce qu'elle est malade et, finalement, il y a ceux qui aiment leur femme et ont une sexualité intéressante avec elle mais qui souhaitent une diversité. Ainsi, certains ont recours aux services d'escorte afin de combler un manque, alors que, pour d'autres, il s'agit surtout de vivre un bon moment en agréable compagnie. Quant à l'échange sexuel lui-même, il peut être unilatéral, le client souhaitant que l'escorte prenne la rencontre en charge de manière à ce qu'il puisse s'abandonner à ses bons soins, heureux de n'avoir rien à faire. Il peut également se présenter comme un désir de partage, dans lequel le client souhaite tout autant donner du plaisir sexuel à sa partenaire du moment qu'à en recevoir. Plusieurs clients, surtout parmi les plus de 40 ans, savent - ou croient savoir - donner un cunnilingus et sont heureux lorsque l'escorte en retire plaisir et orgasmes, leur propre plaisir étant subordonné à celui de la partenaire.

De l'avis répété de plusieurs participantes, la plupart des clients sont des hommes ayant besoin d'intimité affective. Ils ont besoin de tendresse, de se faire toucher, de se confier à quelqu'un qui les écoute, de vivre un moment d'intimité avec une autre personne, de se sentir aimés pour ce qu'ils sont et valorisés. C'est donc dans un état de grande sensibilité et de grande vulnérabilité qu'ils s'ouvrent à l'escorte. Chez eux, le sexuel prend une place limitée dans la rencontre. Ils ont certes besoin d'un moment d'intimité sexuelle et d'un relâchement des tensions, mais, le plus souvent, les activités sexuelles ne durent que de 5 à 15 minutes dans une rencontre d'une heure. Le reste du temps est consacré à un échange verbal dans lequel ils « ouvrent leur cœur » et se permettent de raconter leurs inquiétudes dans un espace où ils s'attendent à être entendus sans être jugés, ou encore 
partagent leur expérience de vie tout en invitant l'escorte à partager également la sienne. Ils veulent une " girl friend experience », c'est-à-dire une rencontre qui ressemble à ce qu'ils pourraient vivre avec une petite amie de cœur. Lorsque l'escorte offre un tel espace de partage de moments d'intimité (ce qui est le cas de plus de la moitié des participantes), ces clients reviennent régulièrement la voir, construisant ainsi un lien d'amitié durable dans le temps, bien qu'à l'intérieur des limites inhérentes à la nature professionnelle de la relation.

Par ailleurs, certains clients viennent voir une escorte «pour la baise » et ne souhaitent pas prendre du temps pour échanger verbalement. Évitant d'emblée ce genre de rencontre, Candy et Gaelle ont été agréablement surprises lorsqu'elles ont eu de tels clients, car, dans chaque cas, ceux-ci se sont montrés respectueux. Bien que l'échange ait été seulement sexuel, le client était attentif à la personne de l'escorte et avait son bien-être à cœur. Une telle attitude respectueuse de la part du client dans les cas où celui-ci souhaite une rencontre axée sur le sexe dans un échange verbal réduit au minimum, a également été rapportée comme habituelle par la plupart des participantes. Néanmoins, dans d'autres cas moins fréquents, le client veut du sexe très intense sur un mode « rough » et plus « animal », ou encore une pénétration qui s'éternise sur presque toute l'heure. Son désir d'une sexualité intense l'amène à oublier que l'escorte peut ne pas avoir l'énergie et la capacité de soutenir le rythme, à oublier que le geste si excitant en fantasme (gorge profonde ou pénétration en coup de bélier, par exemple) est, en fait, très inconfortable, voire même douloureux, dans la réalité (Anna, Dona).

Noémi, de son côté, s'est spécialisée dans l'offre d'une «porn star experience », ce qui lui permet de répondre à cette demande de sexualité plus «hard core » qu'ont certains clients, sans se sentir dévalorisée. Tout ce que l'on voit dans le film porno est alors offert au client, dans l'ordre du raisonnable et avec l'exigence du port du condom pour la pénétration vaginale ou anale. Il s'agit donc de « baisers, caresses, masturbation, fellation, cunnilingus, relation vaginale protégée, relation anale protégée, anulinctus, deep throat, cumshot facial, soft domination, soft soumission, spanking, gagging... ». Noémi voit en outre à se préparer (dilatation anale), avant la rencontre, afin d'être en mesure de recevoir la pénétration anale qui peut parfois être faite sans interruption et très sauvagement. Il est à noter que le tarif de ce type de service est de deux à trois fois celui d'une rencontre régulière et que Noémi trouve un véritable plaisir à performer ce jeu théâtral de la porn star.

Finalement, un nombre non négligeable de clients rencontrent une escorte afin de jouer un fantasme sexuel autre que pornographique, car ils ne peuvent le faire avec leur partenaire, soit parce que cela ne s'y prête pas ou encore parce que celle-ci ne partage pas le fantasme en question. Quelques participantes se sont spécialisées dans les demandes de fétichisme et de domination et reçoivent ce type de client. Les autres participantes rapportent avoir parfois reçu des demandes de jeu de rôle, soulignant que ces demandes sont souvent faites dans le plus grand respect de la travailleuse du sexe. Certaines acceptent de répondre à la demande et d'autres non. Gaelle estime « fantastique » cette possibilité qui lui est ainsi offerte d'entrer dans l'univers de personnes ayant des fantasmes différents des siens et d'explorer divers rôles, allant de celui où elle personnifie une secrétaire à celui où elle insulte le client en l'obligeant à «être un bol de toilette » et en lui urinant dans la bouche. Quelques expériences avec une personne fétichiste l'ont par ailleurs amenée à prendre conscience de la beauté et de l'érotisme du pied. C'est donc avec enthousiasme qu'elle en remercie le client, tout en soulignant qu'elle n'est elle-même toutefois pas devenue fétichiste pour autant. 
Maryse et Irina ont, chacune de leur côté, rapporté quelques situations où un client voulait jouer un scénario dans lequel ce dernier insulte l'escorte lors de l'acte sexuel. À chaque fois, celui-ci a expliqué par avance ce qu'il souhaitait faire et a demandé l'assentiment de la travailleuse. Après la rencontre, le client s'est confondu en excuses, soulignant qu'il ne s'agissait que d'un scénario et non pas de ce qu'il pensait vraiment d'elle comme personne ou comme travailleuse. Parce qu'elles avaient été prévenues et qu'elles avaient accepté de jouer le scénario, elles ne se sont pas senties diminuées par un tel jeu de rôle.

Dans d'autres situations, toutefois, le client impose son fantasme à l'escorte, amenant celle-ci à se sentir mal à l'aise ou agressée. Par exemple, Heidi a connu quelques clients qui, par leur manière d'agir en la traitant de «pute » avec des «Avoue-le, tu aimes ça mon argent! », l'ont amenée à se sentir objet sexuel. Pour Évelyn, ce fut un client qui s'est mis à la malmener, croyant, semble-t-il, qu'elle appréciait les activités sado-masochistes; il a toutefois arrêté quand elle lui a dit qu'elle n'aimait vraiment pas ça. Quelques participantes ont rencontré un client leur demandant, au contraire, de prendre un rôle de dominante, mais sans l'avoir annoncé d'avance. Celles qui sont à l'aise de jouer ce rôle n'ont pas eu de difficulté. Par contre, les autres se sont senties mal à l'aise ou incompétentes face à cette demande imprévue. Océane, de son côté, a reçu un client qui lui a demandé d'avoir des activités sexuelles avec son chien à elle. Même si elle a refusé catégoriquement, cela l'a bouleversée pendant longtemps, d'autant plus que le client la harcelait en la rappelant régulièrement dans le but d'obtenir la satisfaction de son fantasme. Elle n'a finalement pu s'en débarrasser qu'en acceptant de le voir (chez elle) pour 200\$. Après qu'il ait payé, elle lui a réitéré son refus d'avoir quoi que ce soit de sexuel avec son chien et lui a dit qu'elle ne voulait plus jamais le revoir chez elle. Il a finalement compris et est reparti sans chercher à la violenter.

Plusieurs participantes ont remarqué qu'une majorité de clients sont tout autant intéressés et attirés par leur personnalité que par leurs attributs physiques, et que pour faire de l'escorte, il n'est pas nécessaire de se conformer à un modèle prédéfini et stéréotypé. Ainsi, Béatrice raconte comment un client «n'en revient pas » de l'aimer, alors qu'elle n'est pas du tout physiquement de son genre. Irina, qui a 45 ans et un « corps de fermière » en forme, est convaincue que ses clients viennent la voir pour sa personnalité et non pas pour sa beauté. Anna a remarqué qu'une de ses collègues avec qui elle fait des duos, maintient une bonne clientèle alors qu'elle ne prend pas soin de sa coiffure, arrivant « toute couettée » chez le client, et qu'elle ne se rase ni les jambes ni les aisselles. Dans le même sens, Dona fait remarquer qu'une escorte moins jolie, mais qui possède à la fois beauté intérieure, charisme, intelligence et capacité de soutenir une conversation intéressante, réussira mieux à construire et maintenir une bonne clientèle qu'une femme très belle, mais qui ne possède pas ces qualités. Maryse, de son côté, ne se maquille jamais; ce qui ne l'empêche pas d'avoir sa clientèle tout autant dans la danse érotique, que dans le massage ou le service d'escorte. Enfin, Noémi constitue un exemple de ce qu'une femme peut ne pas être mince et être pourtant très demandée, celle-ci pesant $100 \mathrm{~kg}$ pour une hauteur de $1 \mathrm{~m} \mathrm{65}$. Ces observations montrent que beaucoup de clients ne réduisent pas les escortes à leur corps et à leur sexe. Au contraire, ils reconnaissent la valeur humaine de l'escorte et, sensibles à celle-ci, ils souhaitent établir avec l'escorte une relation qui inclut des échanges intellectuels, émotionnels et affectifs. 


\section{b) Des rencontres généralement sous le signe du respect}

À l'exception de Flora, toutes les participantes ont souligné combien la grande majorité de leurs clients sont respectueux et gentils, font preuve d'une attitude réceptive à leur égard et respectent leurs limites quant aux actes sexuels possibles. Selon Joane, les clients veulent au minimum que l'escorte se sente bien lorsqu'elle est avec eux, et si elle a du plaisir sexuel, ils en sont encore plus contents. Anna dit d'eux qu'ils l'écoutent, font attention à elle, sont doux dans leur approche et leurs caresses, sont sexuellement généreux et veulent lui donner du plaisir. Béatrice voit ses clients comme des «amis » avec qui elle a à la fois un échange verbal intéressant et un échange sexuel agréable. Noémi affirme avoir toujours eu de bons clients, des gens très réceptifs et respectueux, qui lui demandent ses limites et les respectent. À l'occasion, certains insistent toutefois, mais ils le font alors sans rien forcer. Joane, de son côté, avait observé que la culture compte pour beaucoup en ce qui concerne le degré de respect envers l'escorte et que les anglophones et les Américains tendent à positionner tout simplement l'escorte sur un piédestal. Béatrice préfère les Américains pour cette même raison. Quant à Dona, elle s'est toujours sentie fascinée par le client qui sait bien toucher et se soucie de lui procurer du plaisir dans une sorte d'échange mutuel, chose assez fréquente pour elle. Irina, par ailleurs, s'est constitué un harem de clients-amants qui l'apprécient et lui rendent différents services en dehors des heures «de travail », c'est-à-dire en dehors des moments d'intimité sexuelle. Finalement, Paola, qui est mal à l'aise de donner le service sexuel pour lequel elle est payée, a noté que la plupart des clients respectent généralement ses limites. Cellesci sont pourtant grandes, car elle refuse la pénétration avec le client qu'elle voit la première fois. Autre détail intéressant, les quelques participantes ayant dit à leur premier client que c'était leur première fois, ont été agréablement surprises par l'attitude très compréhensive et empreinte de délicatesse de leur part, ceux-ci cherchant dès lors à rendre la rencontre aussi agréable que possible pour elles.

C'est donc dire que la plupart des clients se présentent et agissent en ami envers l'escorte. Loin de percevoir celle-ci comme un vulgaire morceau de viande dans lequel se vider, ils veulent une véritable relation, même s'il s'agit d'une relation limitée dans le temps. C'est ainsi qu'au-delà du désir sexuel à assouvir, les clients tiennent habituellement compte de la personne qu'est l'escorte et lui veulent du bien. Cependant, ce ne sont pas tous les clients qui présentent cette attitude respectueuse et il arrive parfois que certains sont montrent tout à fait désagréables, voire violents.

\section{c) Quelques clients désagréables ou violents}

La grande majorité des participantes n'ont jamais été violentées physiquement ou forcées à faire un acte sexuel qu'elles refusaient, mais la plupart ont parfois rencontré des clients désagréables et quelques-unes ont rencontré un client violent. Vulnérabilisée par une toxicomanie, seule Flora a souvent rencontré des clients qui ont abusé d'elle en outrepassant ses limites, en la payant moins qu'entendu au départ ou en faisant preuve de comportement agressif à son égard. Elle souligne toutefois qu'elle en était certainement en partie responsable puisque sa toxicomanie ne lui permettait plus de déterminer ses limites et de les maintenir, ce qui a encouragé différents clients à abuser d'elle. De plus, elle avait développé un «caractère très agressif et impulsif, [ce qui fait que] ça finissait souvent dans des chicanes » avec les clients. Chez les autres participantes, seules Anna, Évelyn, Lisa et Océane ont rencontré un client agresseur. Pour Anna, il s'agit d'un client qui a réussi à enlever le condom juste avant l'éjaculation lors de la pénétration vaginale. Elle a alors senti sa vie 
en danger, ayant très peur d'avoir contracté une ITS ou le VIH. Elle n'a plus été capable de travailler pendant plusieurs jours, la peur l'envahissant à chaque fois. Une consultation médicale et des résultats négatifs l'ont finalement aidée à dépasser cet événement. Ce qui l'a le plus étonnée, c'est qu'ensuite ce client est revenu la voir (en agence) et qu'il ne semblait pas comprendre la colère habitant Anna lorsqu'elle lui a dit qu'elle ne voulait jamais le revoir; pour lui, semble-t-il, il ne s'agissait pas d'un comportement inadmissible. De son côté, Évelyn a, à une occasion, subi la violence d'un client qui la frappait sur les fesses et qui lui répondait « Je t'ai payé, criss de chienne » lorsqu'elle exigeait qu'il arrête. Pour Lisa, il s'agissait d'un client saoul à qui elle avait chargé un très gros montant parce qu'il était tard le soir. Il voulait de l'anal mais Lisa le lui refusait car il ne voulait pas payer extra. Face à son insistance, elle a décidé de quitter avant la fin de l'heure et comme elle refusait également de lui rendre la moitié du montant payé, le client l'a agressée pour reprendre l'argent, puis il l'a poussée hors de l'appartement et en bas des escaliers. Quant à Océane, elle s'est présentée à un rendez-vous où le client, accompagné d'un ami, lui a dit qu'il n'avait pas d'argent. L'ami est sorti pour aller chercher de l'argent et le client en a profité pour la plaquer sur le lit et essayer de la violer. L'ami revenant rapidement à la maison, il a exigé du client qu'il la lâche et la laisse partir. Ces exemples - qui englobent la totalité des agressions rapportées par les 16 participantes - démontrent que l'escorte qui n'a pas été vulnérabilisée par une toxicomanie peut effectivement être victime d'agression, mais il s'agit toutefois d'une expérience rarissime.

À la question de «comment ça se passe quand ça ne se passe pas bien sexuellement avec un client? », plusieurs participantes ont répondu, contre toute attente, que ce sont les rencontres où le client ne se présente pas, car cela leur fait perdre du temps et de l'argent. Ce n'est qu'après être revenues sur la question que, tout en soutenant que cela n'arrive pas souvent, elles traitent de leur expérience des clients désagréables et, encore là, ce n'est pas nécessairement sexuel. Parfois le client n'a aucune intention d'agresser, mais il est ressenti comme agressant par l'escorte parce qu'il est malhabile et que, dans sa maladresse physique et sexuelle, il en devient agaçant et fait perdre patience ou, pire, il fait mal. Parfois, la maladresse se retrouve dans un désir d'aider : " Mais tu es intelligente, tu pourrais faire mieux que ça. Tu es capable de retourner aux études, d'avoir un diplôme, de te trouver un meilleur emploi. La prostitution, ce n'est pas pour les filles intelligentes comme toi ». Loin de s'en sentir valorisée, l'escorte ressent habituellement ces commentaires comme porteurs d'un jugement négatif quant à ses choix. Dans d'autres cas, le client semble prendre pour acquis que l'escorte est là pour répondre à son fantasme sans même qu'il ait à lui en parler avant et à le lui demander, comme si l'escorte, de par son métier, était nécessairement ouverte et consentante à tout. Par ailleurs, quelques clients sont tout à fait arrogants, dénigrants, et sexuellement exigeants, comme si tout leur était dû. Certains insistent pour obtenir ce qu'ils veulent après avoir fait mine d'accepter un premier refus, manipulant physiquement l'escorte pour l'obtenir comme si elle avait déjà accepté (ex., en la positionnant pour une sodomie), ou encore ôtant le condom lors de l'acte sexuel. Ceci oblige l'escorte à demeurer vigilante, à rappeler constamment les limites, mais aussi à les maintenir fermement en agissant à chaque fois promptement pour se protéger. Confirmant cette observation, Sylvie se rappelle avoir vécu plusieurs situations inconfortables et agressantes lorsqu'elle était adolescente et ne possédait pas ces habiletés par manque d'expérience de vie. Elle remarque que ces événements n'ont rien d'une agression « comme on en voit dans les journaux », mais il n'en demeure pas moins que, ignorant qu'elle avait le droit de mettre ses limites et de les faire respecter, elle ne savait pas se protéger contre différentes situations abusives. 


\section{d) Un portrait contrasté du client}

Comme nous avons pu le constater à travers ces différents témoignages présentés par les participantes, les clients rencontrés ne correspondent nullement au stéréotype du client agresseur qui chercherait à imposer violemment sa propre sexualité par pur machisme, usant du corps de la femme comme d'un vulgaire objet dans lequel se vider. Au contraire, la plupart de ces clients sont à la recherche d'un contact mutuellement chaleureux dans lequel ils peuvent répondre à certains de leurs besoins affectifs et sexuels. Adoptant par conséquent un comportement favorisant un contact harmonieux et un échange d'intimité affective, ils sont amenés à respecter l'escorte en tant que personne, même si la relation est tarifée et qu'ils sont là pour leurs propres besoins. Malgré tout, quelques clients s'avèrent désagréables, et, très exceptionnellement, violents.

Par ailleurs, cette description ne correspond pas nécessairement à l'ensemble des clients requérant des services d'escorte, car notre échantillonnage n'est pas représentatif de l'expérience qu'en ont toutes les femmes offrant ces services, celui-ci regroupant essentiellement des femmes confortables à offrir des services sexuels et à discuter ouvertement de leur sexualité. De plus, la plupart de nos participantes ont la possibilité de choisir leurs clients et donc de refuser les hommes ayant une attitude vulgaire ou se montrant moins respectueux. D'ailleurs, l'expérience de Sylvie, lorsqu'elle était mineure, et celle de Flora qui est devenue très vulnérable à l'exploitation et à la violence lorsqu'elle était toxicomane, suggèrent que les proportions de bons et de mauvais clients varient selon les conditions entourant la pratique du travail du sexe et qu'une femme qui n'a pas les ressources nécessaires pour s'affirmer et se protéger est plus susceptible de rencontrer des clients qui lui manqueront de respect. Par contre, notre échantillon englobe quelque 74 ans de travail d'escorte, avec une moyenne de 4,6 ans par participante. Présumant que celles-ci ont rencontré au moins un nouveau client toutes les deux semaines, on peut en déduire qu'ensemble, elles ont rencontré au moins 3,700 clients différents. Même en tenant compte de la petite portion des clients s'avérant désagréables, il demeure qu'un nombre significatif de clients sont tout à fait respectueux et ne correspondent aucunement au stéréotype du prédateur sexuel. Ces données recueillies auprès de femmes qui, recevant des clients requérant leurs services, sont donc les plus à même de témoigner de l'expérience qu'elles en ont, indiquent que les clients n'ont pas nécessairement un comportement répréhensible, bien au contraire. Nos résultats recoupent également ceux provenant d'un bon nombre d'autres études, dont ceux cités en introduction de cet article. D'autre part, la plupart des participantes apprécient le contact chaleureux qu'elles ont avec ces clients et elles sont contentes de répondre aux besoins de ces derniers dans un cadre professionnel où elles sont rémunérées, ce qui contredit également le stéréotype de la femme non consentante, abusée et, finalement, exploitée.

\section{Conclusion}

En regard de ces résultats, nous devons soutenir la décriminalisation du travail du sexe et une noncriminalisation des clients puisque, d'une part, criminaliser les clients ne rendrait nullement justice aux personnes travailleuses du sexe et, d'autre part, cela créerait une condition d'injustice vis-à-vis d'hommes au comportement tout à fait adéquat et qui s'adressent à des femmes consentantes et à l'aise de répondre à leurs besoins affectifs et sexuels en échange d'une rémunération. Ainsi que le démontre Corriveau (2010), le Code criminel canadien contient toutes les lois nécessaires pour 
contrer les situations de violence, de coercition, d'exploitation et de trafic humain. Il n'est donc pas utile de mettre en place des lois spécifiques au travail du sexe. Par contre, il y a place pour amélioration, car bien que le nombre de clients désagréables rencontrés par nos participantes soit minime, il est possiblement plus grand chez les travailleuses du sexe socioéconomiquement plus vulnérables. Outiller ces dernières afin de leur permettre de mieux gérer les clients arrogants et de faire respecter leurs limites constitue un moyen de le faire. Il serait également utile d'éduquer les clients afin qu'ils apprennent à tenir compte de ce que chaque travailleuse a ses champs d'expertise et ses limites et qu'ils doivent à la fois faire leur choix d'escorte en conséquence et annoncer leur demandes « spéciales » à l'avance, plutôt que de prendre pour acquis qu'une escorte, « ça fait tout ». Finalement, déstigmatiser le travail du sexe permettrait de mieux rejoindre et de répondre aux besoins psychosociaux et de santé des personnes travailleuses du sexe les plus vulnérables, ainsi que le souligne la Commission Mondiale sur le VIH et le Droit (2012), en plus d'améliorer la proportion des clients respectueux envers elles.

\section{Remerciements}

Cette recherche doctorale a été soutenue financièrement par le Fonds de recherche du Québec, Société et Culture (FRQSC), ainsi que par une bourse de soutien au doctorat de l'Université Laval.

\section{A propos de l'auteure}

Jacqueline Comte est candidate au doctorat en sociologie de la sexualité à l'école de service social de l'Université Laval. Elle peut être contactée au comte.jacqueline@gmail.com

\section{Références}

Barry, K. (1979). Female sexual slavery. Englewood Cliffs, NJ: Prentice Hall.

Barry, K. (1995). The prostitution of sexuality. New York: New York University Press.

Bernstein, E. (2007). Temporarily Yours: Intimacy, Authenticity, and the Commerce of Sex. Chicago, IL: University of Chicago Press.

Bernstein, E. (2013). Ce qu'acheter veut dire. Désir, demande et commerce du sexe. Actes de la Recherche en Sciences Sociales. 198, 60-77.

Clouet, E. (2008). La prostitution étudiante à l'heure des nouvelles technologies de communication. Paris : Max Milo Éditions.

Coalition des femmes pour l'abolition de la prostitution (2013). Mémoire présenté à la cour suprême du canada par la coalition des femmes pour l'abolition de la prostitution. http://www.abolitionprostitution.ca/francais/documents-legaux 
Commission Mondiale sur le VIH et le droit. (2012). Risques, Droits et Santé. New York :

Programme des Nations Unies pour le Développement.

Comte, J. (à paraître). Parcours dans le travail du sexe de femmes offrant des services d'escorte.

Corriveau, P. (2010). Réguler le travail du sexe : Entre la victimisation et la liberté de choisir. In Parent, C., Bruckert, C., Corriveau, P., Mensah, M.N., \& Toupin, L. Mais oui c'est un travail! Penser le travail du sexe au-delà de la victimisation, 29-54. Québec: Presses de l'Université du Québec.

Di Nicola, A., Cauduro, A., Lombardi, M., \& P. Ruspini, P. (Eds.) (2009). Prostitution and Human Trafficking. Focus on Clients. New York: Springer.

Dworkin, A. (1979). Pornography: Men possessing women. NewYork: G.P. Putnam.

Dworkin, A. (1993). Prostitution and male supremacy. Michigan Journal of Gender \& Law, 1, 112.

Farley, M. (2003). Prostitution and the Invisibility of Harm. Women \& Therapy, 26(3), 247-280.

Farley, M. (2004). 'Bad for the body, bad for the heart': Prostitution Harms Women Even if Legalized or Decriminalized. Violence Against Women, 10(10), 1087-1125.

Farley, M. (2005). Prostitution harms women even if indoors: Reply to Weitzer. Violence Against Women, 11(7), 950-964.

Farley, M. \& Barkan, H. (1998). Prostitution, Violence, and Posttraumatic Stress Disorder. Women \& Health, 27( 3), 37-49.

Farley, M., Cotton, A., Lynne, J., Zumbeck, S., Spiwak, F., Reyes, M. E., Alvarez, D. \& Sezgin, U. (2004). Prostitution and Trafficking in Nine Countries. Journal of Trauma Practice, 2(3), 33-74.

Farley, M., Bindel, J., \& Golding, J. M. (2009). Men Who Buy Sex: Who They Buy and What They Know. London: Eaves. Retrieved from: http://www.eaves4women.co.uk/Documents/Recent_Reports/MenWhoBuySex.pdf

Holt, T. J. \& Blevins, K. R. (2007). Examining sex work from the client's perspective: assessing johns using on-line data. Deviant Behavior. 28, 333 -354.

Hugues, D. (2005). The demand for victims of sex trafficking. Kingston: University of Rhode Island.

Jeffrey, L. A., \& MacDonald, G. (2006). Sex Workers in the Maritimes Talk Back. Vancouver; Toronto: UBC Press.

Jeffreys, S. (1997). The Idea of Prostitution. North Melbourne, Vic: Spinifex Press. 
Jeffreys, S. (2008). Normalising Prostitution and Trafficking: Language Matters. Labrys, études féministes, janvier/juin.

Jeffreys, S. (2009). The Industrial Vagina: The Political Economy of the Global Sex Trade. Routhledge: New York.

Jordan, J. (1997). User Pays : Why Men Buy Sex. The Australian and New Zealand Journal of Criminology. 30, 55-71.

Joseph, L. J. \& Black, P. (2012). Who's the Man? Fragile Masculinities, Consumer Masculinities, and the Profiles of Sex Work Clients. Men and Masculinities, 15(5), 486-506.

Katsulis, Y. (2010). “'Living Like a King': Conspicuous Consumption, Virtual Communities, and the Social Construction of Paid Sexual Encounters by U.S. Sex Tourists.' Men and Masculinities 13, 210-30.

Kinnell, H. (2006). Clients of Female Sex Workers: Men or Monsters? In Campbell, R. \& O'Neill, M. Sex Work Now, 212-234. UK: Willan Publishing.

Klein, C., M., Kennedy, A, \& Gorzalka, B. B. (2009). Rape Myth Acceptance in Men Who Completed the Prostitution Offender Program of British Columbia. International Journal of Offender Therapy and Comparative Criminology. 53(3), 305-315.

Lever, J., \& Dolnic, D. 2010. "Call Girls and Street Prostitutes: Selling Sex and Intimacy." In Sex for Sale: Prostitution, Pornography, and the Sex Industry, edited by R. Weitzer, $2^{\text {nd }}$ ed., 187-203. New York: Routledge.

Lowman, J. \& Atchison, C. (2006). Men Who Buy Sex: A Survey in the Greater Vancouver Regional District. The Canadian Review of Sociology and Anthropology. 43(3), 281-296.

Lucas, A. M. (2005). The Work of Sex Work: Elite Prostitutes' Vocational Orientations and Experiences. Deviant Behavior, 26, 513-546.

MacKinnon, C. A. (1985). Pornography, civil rights, and speech. Harvard Civil Rights-Civil Liberties. Law Review, 20, 1-70.

Milrod, C. \& Monto, M. A. (2012). The Hobbyist and the Girlfriend Experience: Behaviors and Preferences of Male Customers of Internet Sexual Service Providers, Deviant Behavior, 33(10), 792-810.

Milrod, C. \& Weitzer, R. (2012). The Intimacy Prism: Emotion Management among the Clients of Escorts. Men \& Masculinities, 15(5), 447-467.

Monto, M. A. (2000a). “Why men seek prostitutes.' In Sex for Sale. Prostitution, Pornography and the Sex Industry, edited by R. Weitzer, 67-85. New York: Routledge. 
Monto, M. A. (2000b). Focusing on the Clients of Street Prostitutes: A Creative Approach to Reducing Violence Against Women; Summary Report. Washington, DC: National Institute of Justice. https://www.ncjrs.gov/pdffiles1/nij/grants/182859.pdf

Monto M. A. (2004). Female prostitution, Customers and Violence. Violence against Women, 10, 160-168.

Monto, M. (2010). “Prostitutes' Customers: Motives and Misconceptions.' In Sex for Sale: Prostitution, Pornography, and the Sex Industry, edited by R.Weitzer. $2^{\text {nd }}$ ed, 233-254. New York: Routledge.

Monto M. A. \& Hotaling, N. (2001). Predictors of rape myth acceptance among male clients of female sex street prostitutes. Violence against Women, 7, 275-293.

Monto, M. \& Milrod, C. (2013). Ordinary or Peculiar Men? Comparing the Customers of Prostitutes With a Nationally Representative Sample of Men. Int J Offender Ther Comp Criminol. 58(7), 802-820.

Monto, M. A. \& McRee, N. (2005). “A Comparison of the Male Customers of Female Street Prostitutes with National Samples of Men." Int J Offender Ther Comp Criminol, 49:505-29.

Oerton, S., \& Phoenix, J. (2001). Sex-bodywork: Discourses and Practices. Sexualities. 4(4), 387412.

Orchard, T., Farr, S., Macphail, S., Wender, C., \& Young, D. (2012). Sex Work in the Forest City: Experiences of Sex Work Beginnings, Types and Clientele Among Women in London, Ontario. Sex Res Soc Policy, 9, 350-362.

Parent, C., \& Bruckert, C. (2005). Le travail du sexe dans les établissements de services érotiques: une forme de travail marginalisé. Déviance et Société. 29(1), 33-53.

Pitts, M. K., Smith, A. M. A., Grierson, J., O'Brien, M., \& Misson, S. (2004). Who Pays for Sex and Why? An Analysis of Social and Motivational Factors Associated With Males Clients of Sex Workers. Arch Sex Behav. 33(4), 353-358.

Prasad, M. (1999). "'The Morality of Market Exchange: Love, Money and Contractual Justice.' Sociological Perspectives. 42(2), 181-214.

Poulin, R. (2004). La mondialisation des industries du sexe. Prostitution, pornographie, traite des femmes et des enfants. Ottawa: Les Éditions L'Interligne.

Raphael, J., \& Shapiro, D. L. (2002). Sisters Speak Out: The Lives and Needs of Prostituted Women in Chicago; A Research Study. Chicago: Center for Impact Research. Retrieved from http://www.impactresearch.org/documents/sistersspeakout.pdf 
Raphael, J., \& Shapiro, D. (2004). Violence in indoor and outdoor prostitution venues. Violence Against Women. 10, 126-139.

Raymond, J. G. (2003). «Ten Reasons for Not Legalizing Prostitution and a Legal Response to the Demand for Prostitution », in M. Farley (dir.), Prostitution, Trafficking, and Traumatic Stress, Bringhampton: The Haworth Press, pp. 315-332.

Raymond, J. G. (2004). Prostitution on Demand: Legalizing the Buyers as Sexual Consumers. Violence Against Women. 10, 1156-1186.

Sanders, T. (2008). Male Sexual Scripts: Intimacy, Sexuality and Pleasure in the Purchase of Commercial Sex. Sociology. 42(3), 400-417.

Sharp, K., \& Earle, S. 2002. "Cyberpunters and Cyberwhores: Prostitution on the Internet.' In Dot.cons: Crime, Deviance, and Identity on the Internet, edited by Y. Jewkes, 36-52. Uffculme: Willan.

Serughetti, G. (2012). Prostitution and Clients' Responsibility. Men and Masculinities, 16(1), 3548.

Waltman, M. (2011). Sweden's prohibition of purchase of sex: The law's reasons, impact, and potential. Women's Studies International Forum. 34, 449-474.

Welzer-Lang, D., Barbosa, O., \& Mathieu, L. (1994). Prostitution: les uns, les unes et les autres. Paris: Éditions Métailié.

Xantidis, L. \& \& McCabe, M. P. (2000). Personality Characteristics of Male Clients of Female Commercial Sex Workers in Australia. Arch Sex Behav, 29(2), 165-176. 